\title{
Tracheostomy time-out: a new safety tool in the setting of COVID-19
}

\author{
Harish Dharmarajan, M.D. ${ }^{1}$ \\ ${ }^{1}$ University of Pittsburgh Medical Center,
}

April 27, 2020

\begin{abstract}
Tracheostomy procedures have a high risk of aerosol generation. Airway providers have reflected on ways to mitigate the SARSCoV-2 transmission risks when approaching a surgical airway. To standardize institutional safety measures with tracheostomy, we advocate using a dedicated tracheostomy time-out applicable to all patients including those suspected of having COVID-19. The aim of the tracheostomy time-out is to reduce preventable errors that may increase the risk of transmission of SARS-CoV-2.
\end{abstract}

\section{Harish Dharmarajan, M.D. ${ }^{1}$, Carl H. Snyderman, M.D. ${ }^{1}$}

${ }^{1}$ Department of Otolaryngology, University of Pittsburgh Medical Center, Pittsburgh, PA

\section{Corresponding Author :}

Harish Dharmarajan, M.D.

Department of Otolaryngology, UPMC

Eye \& Ear Institute, Suite 500

200 Lothrop Street

Pittsburgh, PA 15213

Email: dharmarajanh@upmc.edu. Phone: 3107365675.

Funding : There were no sources of funding for this project.

Prior Presentations : This project has not been presented or published in prior meetings.

Running Title : Tracheostomy time-out

Key Words : tracheostomy, COVID-19, time-out, safety, airway

\section{Abstract}

Tracheostomy procedures have a high risk of aerosol generation. Airway providers have reflected on ways to mitigate the SARS-CoV-2 transmission risks when approaching a surgical airway. To standardize institutional safety measures with tracheostomy, we advocate using a dedicated tracheostomy time-out applicable to all patients including those suspected of having COVID-19. The aim of the tracheostomy time-out is to reduce preventable errors that may increase the risk of transmission of SARS-CoV-2.

\section{Background}


The SARS-CoV-2 virus (severe acute respiratory syndrome coronavirus 2) which causes COVID-19 infection (coronavirus disease 2019) has dramatically changed the way that physicians approach airway procedures. SARS-CoV-2 affects multiple levels of the aerodigestive tract. Viral loads in the lower respiratory tract samples (sputum) appear to be significantly higher compared to those from nasal or throat swabs ${ }^{1}$. Although the exact route of transmission is not well defined, SARS-CoV-2 is thought to be spread via a combination of contact, droplet, and airborne routes ${ }^{2}$. The Centers for Disease Control and Prevention has recommended use of personal protective equipment for both patients and healthcare personnel in order to decrease risk of transmission; this entails that patients use facemasks and providers utilize isolation gowns, gloves, N95 respirators, and face shields or goggles ${ }^{3}$. Commonly performed airway procedures including intubation, direct laryngoscopy, bronchoscopy, and tracheostomy placement have a high risk of aerosol generation. Airway providers have reflected on ways to mitigate the transmission risks especially when approaching a surgical airway. Several academic organizations have outlined new recommendations for tracheostomy placement in the setting of the COVID-19 pandemic ${ }^{4-7}$. To standardize institutional safety measures with tracheostomy, we advocate using a dedicated tracheostomy time-out applicable to all patients including those suspected of having COVID-19 (Figure 1). The aim of this specific tracheostomy time-out is to reduce preventable errors that may increase the risk of transmission of SARS-CoV-2 .

\section{Elements of Transmission Risk Reduction}

In a tracheostomy procedure, risk reduction of SARS-CoV-2 transmission can be broken down into three components: preparation, personnel, and process (Figure 2).

1. Preparation . Patients who undergo an elective tracheostomy should receive pre-operative SARS$\mathrm{CoV}-2$ testing. In emergent airway settings however, this is not feasible. Several days before the procedure, the operating room or ICU coordinator must ensure that a negative pressure room is available and that appropriate personal protective equipment will be available for the entire OR staff. Before the procedure is started, we advocate that a dedicated tracheostomy time-out should be conducted. The time-out functions as a concise, standardized briefing between the Otolaryngology and Anesthesia teams as well as the rest of operative room staff. Key aspects of the case should be discussed at that moment including operative plan, paralytic and reversal plans, and expected sequences for holding ventilation or changing circuits. When the surgical team is close to entering the airway, there should again be a discussion with the anesthesia team regarding the expected next steps. A part of the tracheostomy time-out is focused on intra-operative safety measures (Figure 1, light magenta background); this portion of the time-out should be repeated when close to entering the airway.

2. Personnel . The surgical team personnel should be proficient at donning and removing PPE carefully. It is recommended that a spotter be assigned to ensure that providers are properly wearing PPE and do not accidently contaminate themselves while removing the PPE. Only the needed personnel should be present for the tracheostomy. Teamwork is essential, and open communication must be encouraged between all staff members during the procedure: surgeons, anesthesiologists, nursing staff, and surgical technologists. Personnel must feel comfortable with bringing forward any findings of error or mistakes. This is expected with any proper safety culture and climate.

3. Process . Both the surgical and anesthesiology teams must take steps to reduce the aerosolization of respiratory secretions during the case. One must also ensure that the ventilator does not become contaminated. Whenever the respiratory circuit is open, ventilation should be held. This is especially important after the tracheotomy incision is made. Ventilation should be held for the following sequence: endotracheal tube (ETT) advancement distally before tracheal incision, tracheal incision and withdrawal of ETT, tracheostomy tube insertion and cuff inflation, ventilation tubing attachment and closing of circuit. If ventilation is needed during this sequence, the surgical and anesthesiology teams should ensure that the cuff of the ETT or tracheostomy tube is first inflated. With any circuit changes, the anesthesia team clamps the ETT before reconnecting the circuit. Suctioning should be limited once the tracheotomy is made. If a bronchoscopy is needed after tracheostomy tube placement, we recommend using a side port and holding ventilation. A two-filter system is suggested by the Anesthesia Patient Safety Foundation; this involves using a viral filter at the ETT (or tracheostomy 
tube) and another at the expiratory limb of the ventilator circuit to prevent machine contamination ${ }^{8}$. Additionally, a filter may be placed at the inspiratory circuit limb if there is concern that the machine itself may become contaminated in-between patient use. Permitting availability, we recommend that viral filters be placed on the patient's airway, ETT or tracheostomy tube, as well as both limbs of the respiratory circuit, inspiratory and expiratory. When transitioning from the ETT to tracheostomy circuit, the contaminated ETT viral filter should be discarded and a new filter should be placed at the tracheostomy tube. Once the patient can be weaned off the ventilator, an HME-filter should be used rather than leaving the tracheostomy tube open to trach mask.

\section{Tracheostomy Protocol at UPMC}

At the University of Pittsburgh Medical Center (UPMC), tracheostomy placement in COVID-19 patients is considered only after 2-3 weeks of mechanical ventilation unless there are compelling clinical reasons necessitating earlier timing. All patients undergoing elective tracheostomy receive pre-operative SARS-CoV2 testing. As testing capacity increases, retesting prior to the procedure is recommended. The procedure should be performed in a negative pressure room for patients who are COVID-19 positive or have an unknown status. Open surgical tracheostomy is preferred to percutaneous dilation approach in order to reduce the risk of aerosolization. The procedure is to be performed in the ICU whenever possible to reduce exposure to staff and other patients. Full PPE with N95 or powered air-purifying respirator (PAPR) is required for all staff in the procedure room. The entire procedure is done with complete paralysis and a high-flow suction device is recommended whenever cautery is used. Ventilation is held when the respiratory circuit is open. Tracheal suctioning is minimized and a cuffed, non-fenestrated tracheostomy tube is always placed.

\section{Survey of UPMC Tracheostomy Providers}

A brief survey focusing on tracheostomy practices during March and April 2020 was completed by Otolaryngologists, General Surgeons, and Thoracic Surgeons at the UPMC Oakland campus. Providers were asked to respond how often certain practices were being used during the procedure: always, usually, about half the time, seldom, or never (Figure 3). In addition, providers were asked to provide suggestions for tracheostomy time-out design especially with regards to key safety measures. A total of 15 providers responded of which 9 had performed tracheostomies in this timeframe (6 Otolaryngologists, 2 General Surgeons, and 1 Thoracic Surgeon). Each provider performed an average of 3 tracheostomies (range: 1-6). None of the patients had confirmed COVID-19 infection although four patients were under investigation at time of procedure. Most providers $(77 \% ; 7 / 9)$ performed open tracheostomies compared to percutaneous approach. On average, two members of the surgical team were present for the procedure (range: 1-3). Only one of the providers noted using a negative pressure room for the tracheostomy. Most providers reported always using the following practices: wearing an N95 mask $(66 \%, 6 / 9)$, patient paralysis $(66 \%, 6 / 9)$, holding ventilation with open respiratory circuit $(77 \%, 7 / 9)$, and using cuffed tracheostomy tube $(88 \%, 8 / 9)$. Only three providers noted waiting for COVID-19 status before performing the tracheostomy tube change. Providers frequently performed the trach change between post-op days 5 to $10(38 \%, 3 / 8)$ or after 10 days $(38 \%, 3 / 8)$; only 2 providers performed the trach change on post-op day 5.

\section{Tracheostomy Timeout Design and Feedback}

Airway providers provided various suggestions for tracheostomy time-out design with a common emphasis on the following topics: COVID-19 status verification, confirming PPE and equipment availability, coordination with anesthesia team, paralytic and reversal plans, electrocautery settings and FiO2 levels near airway, confirming anticipated periods of holding ventilation with open circuit, avoiding tracheal suctioning after tracheotomy, and use of viral filters for ventilator circuit, ETT, and tracheostomy tube. These recommendations were used to create a specific timeout targeting tracheostomy safety measures (Figure 1). The tracheostomy time-out is conducted by the surgical team immediately after completing the general surgical timeout. The middle portion of the time-out, highlighted in the colored background, emphasizes intra-operative safety measures to reduce risk of aerosolization and airway fire; this portion is repeated when the surgical team is close to entering the airway. A hardcopy of the time-out should available in the operating 
room and be posted in a common area where both surgical and anesthesiology teams can have access (i.e. side of anesthesia workstation). Team debriefing is encouraged at the end of the case to review what went well and assess for areas of improvement. The draft of the time-out was sent to a total of 102 physicians for feedback: a mix of Otolaryngologists, General Surgeons, Thoracic Surgeons, and Anesthesiologists. There was an overall positive reception to the idea of implementing a tracheostomy time-out. One provider noted that the wording "time-out" may lead to confusion with the general surgical time-out. In order to prevent any confusion, we added an opening statement emphasizing that the tracheostomy time-out should follow the completion of the general time-out. With continued use, we hope that providers can easily integrate the tracheostomy time-out as an extension of the general surgical time-out.

\section{Implications for Patient and Provider Safety}

The origins of the surgical time-out can be traced back to safety checklists used in the aeronautic industry ${ }^{9}$. The philosophy of usage influences how timeouts or checklists are conducted ${ }^{10}$. A procedural time-out may function as a to-do list or as a backup process to verify tasks completed from memory. The time-out is designed to protect against errors from memory recall. There is usually a component of mutual redundancy in conducting a time-out as multiple team members can monitor each other's responses ${ }^{10}$. Taking cues from other high reliability organizations, the World Health Organization designed a surgical safety checklist which resulted in reducing patient morbidity, mortality, and complication rates ${ }^{11,12}$. In the setting of the COVID-19 pandemic, several academic organizations have released guidelines on performing a tracheostomy. These recommendations are aimed at safety measures to mitigate transmission risk of SARS-CoV-2. Given the potential consequences for error, these standardized operating practices for tracheostomy should not be carried out simply from memory recall. Use of a dedicated tracheostomy time-out will allow for safety measures to be verified in a consistent manner. There are several key advantages to using the tracheostomy time-out: review of critical steps by multiple team members, improvement in communication between OR personnel, and an emphasis on quality control. Disadvantages include possible time delays and checklist fatigue. In order to address this, we have made the tracheostomy time-out focused and concise. If performed without interruptions, the time-out can be completed in under 1 minute.

Next Steps

In the coming weeks, we will widely implement this timeout process at the UPMC Oakland campus. Adherence rates and provider feedback will be tracked. In addition, the OR staff will observe several sessions of the tracheostomy time-out by different providers to determine if any adjustments can be made for ease of use. We will determine which parts of the time-out are deemed important and if any parts appear nonessential. A revised version will then be created and sent to providers again for feedback.

\section{References}

1. Yu F, Yan L, Wang N, et al. Quantitative Detection and Viral Load Analysis of SARS-CoV-2 in Infected Patients. Clinical Infectious Diseases . 2020. doi:10.1093/cid/ciaa345

2. Bahl P, Doolan C, Silva CD, Chughtai AA, Bourouiba L, Macintyre CR. Airborne or droplet precautions for health workers treating COVID-19? The Journal of Infectious Diseases . 2020. doi:10.1093/infdis/jiaa189

3. Using Personal Protective Equipment (PPE). Centers for Disease Control and Prevention. https://www.cdc.gov/coronar ncov/hcp/using-ppe.html. Published April 3, 2020. Accessed April 20, 2020.

4. Parker N, Schiff B, Fritz M, et al. Tracheotomy Recommendations During the COVID-19 Pandemic. American Academy of Otolaryngology-Head and Neck Surgery. https://www.entnet.org/content/tracheotomyrecommendations-during-covid-19-pandemic. Published April 2, 2020. Accessed April 21, 2020.

5. Harrison L, Ramsden J, et al. Tracheostomy guidance during the COVID-19 Pandemic. https://www.entuk.org/tracheo guidance-during-covid-19-pandemic. Accessed April 21, 2020.

6. Michetti CP, Burlew CC, Bulger EM, Davis KA, Spain DA. Performing tracheostomy during the Covid-19 pandemic: guidance and recommendations from the Critical Care and Acute Care Surgery Committees of the American Association for the Surgery of Trauma. Trauma Surgery $\&$ Acute Care Open . 2020;5(1). doi:10.1136/tsaco-2020-000482 
7. Schultz P, Morvan J-B, Fakhry N, et al. French consensus regarding precautions during tracheostomy and post-tracheostomy care in the context of COVID-19 pandemic. European Annals of Otorhinolaryngology, Head and Neck Diseases . 2020. doi:10.1016/j.anorl.2020.04.006

8. Feldman J, Loeb R, Philip J. FAQ on Anesthesia Machine Use, Protection, and Decontamination During the COVID-19 Pandemic. Anesthesia Patient Safety Foundation. https://www.apsf.org/faqon-anesthesia-machine-use-protection-and-decontamination-during-the-covid-19-pandemic/. Accessed April 21, 2020.

9. Newkirk JD. Preventing Surgical Mishaps. Clinics in Plastic Surgery . 2013;40(3):475-487. doi:10.1016/j.cps.2013.04.011

10. Degani A, Wiener EL. Cockpit Checklists: Concepts, Design, and Use. Human Factors: The Journal of the Human Factors and Ergonomics Society . 1993;35(2):345-359. doi:10.1177/001872089303500209

11. Weiser TG, Haynes AB, Lashoher A, et al. Perspectives in quality: designing the WHO Surgical Safety Checklist. International Journal for Quality in Health Care . 2010;22(5):365-370. doi:10.1093/intqhc/mzq039

12. Haynes AB, Weiser TG, Berry WR, et al. A Surgical Safety Checklist to Reduce Morbidity and Mortality in a Global Population. New England Journal of Medicine . 2009;360(5):491-499. doi:10.1056/nejmsa0810119 


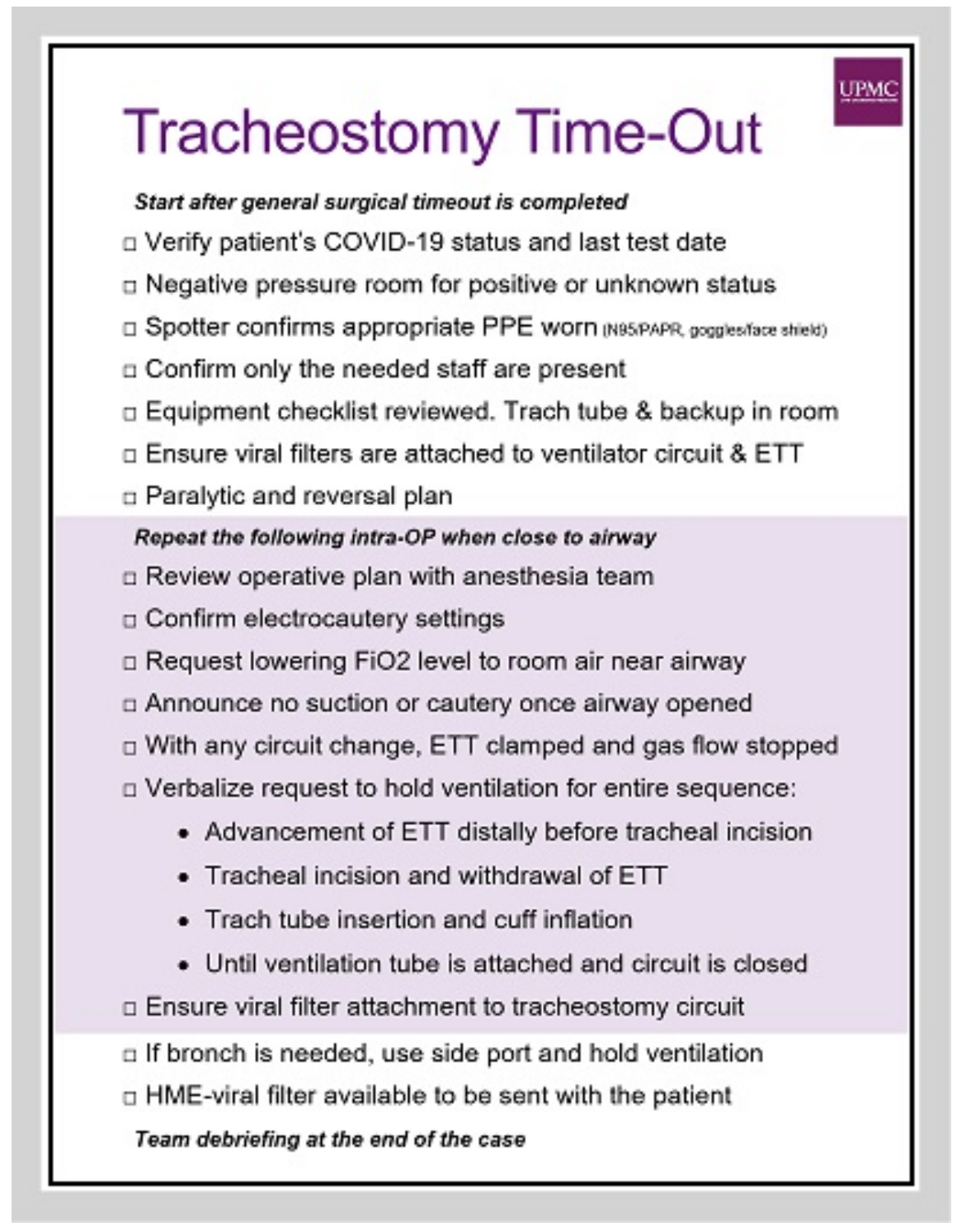

Figure 1: This is a caption

Figure 1. Tracheostomy time-out. This is performed immediately after the general surgical time-out is completed. The middle of the checklist highlighted in light magenta background involves intraoperative safety measures which should be repeated when the surgical team is close to entering the airway. PPE: personal protective equipment. ETT: endotracheal tube. FiO2: fraction of inspired oxygen via anesthesia circuit. HME: heat moisture exchanger. 


\begin{tabular}{|c|c|}
\hline \multicolumn{2}{|r|}{ Risk Reduction Measures } \\
\hline 흘 & $\begin{array}{l}\text { COVID-19 testing pre-OP } \\
\text { Operative room setup } \\
\text { PPE request } \\
\text { Tracheostomy time-out }\end{array}$ \\
\hline 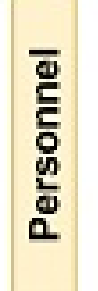 & $\begin{array}{l}\text { PPE donning \& removal } \\
\text { Only needed staff in the OR } \\
\text { Safety culture } \\
\text { Safety climate in OR }\end{array}$ \\
\hline ฏ & $\begin{array}{l}\text { Viral filters for ETT, ventilator, and trach } \\
\text { Lowering } \mathrm{FiO} 2 \text { levels near airway } \\
\text { Electrocautery settings } \\
\text { Reduced suctioning once airway opened } \\
\text { Sequence of holding ventilation } \\
\text { ETT clamping with circuit changes } \\
\text { HME-filter usage post-op }\end{array}$ \\
\hline
\end{tabular}

Figure 2: This is a caption

Figure 2. Summary of risk reduction measures for performing a tracheostomy. Pre-OP: pre-operative period. PPE: personal protective equipment. ETT: endotracheal tube. FiO2: fraction of inspired oxygen via anesthesia circuit. HME: heat moisture exchanger. 


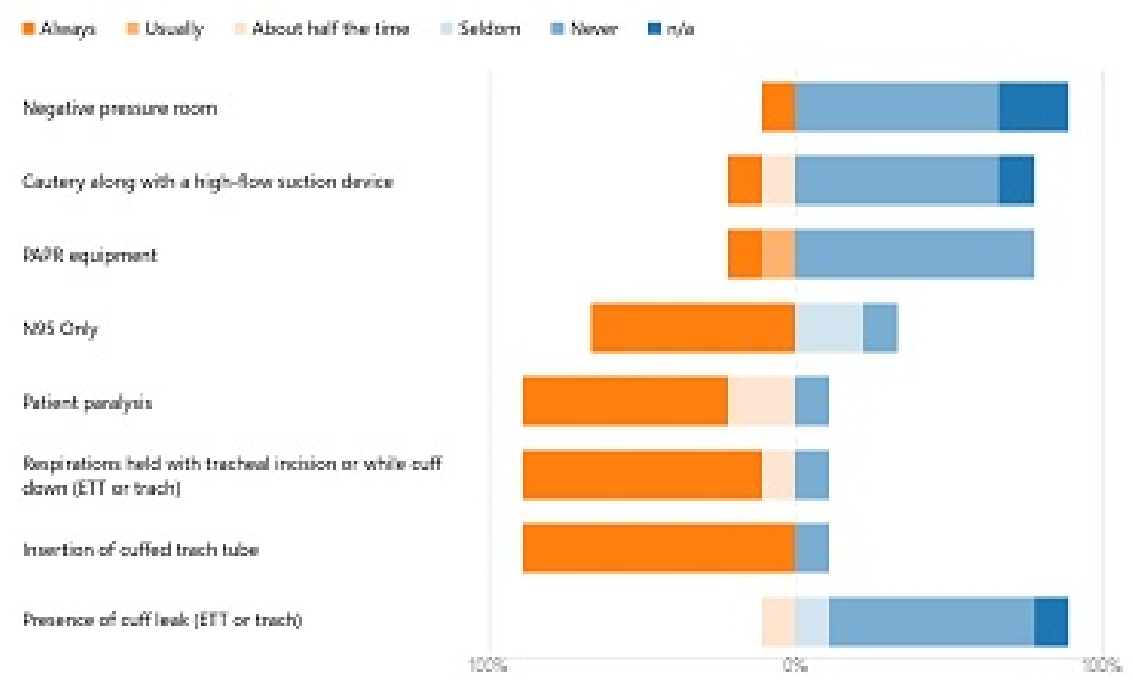

Figure 3: This is a caption

Figure 3 . Provider survey of tracheostomy practices in March and April 2020. Responses are from 9 providers across UPMC Otolaryngology, General Surgery, and Thoracic Surgery. None of the patients had confirmed COVID-19 infection but 4 patients were under investigation at time of procedure. PAPR: powered air-purifying respirator. ETT: endotracheal tube. 